\title{
DFMEA 在高压线束系统设计中的应用
}

\section{Application of DFMEA in the Design of High-Voltage Harness System \\ 郭春洪}

Chunhong Guo

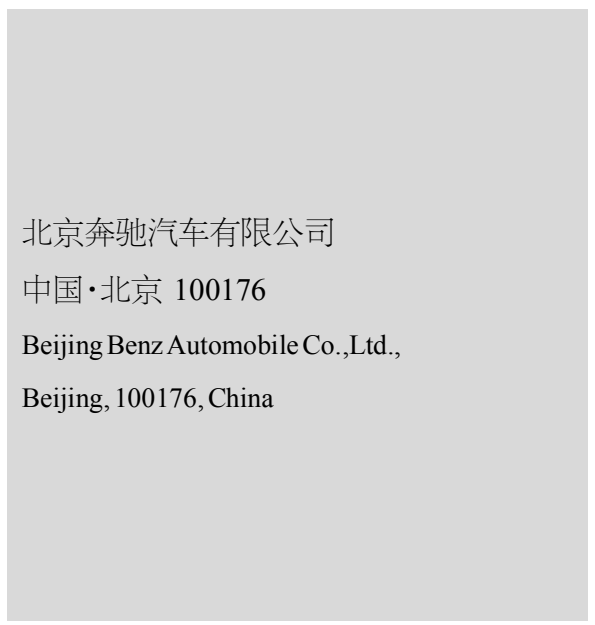

【摘要】论文介绍了新能源高压线束的功能, 从新能源汽车使用特性出发, 分析了新能 源汽车高压线束的设计潜在失效模式及其影响, 并明确 SOD 的判定标准; 对整车高压线 束系统的设计过程控制及产品质量提升有积极意义。

【Abstract】This paper introduces the function of new energy high-voltage harness, analyzes the design potential failure mode and its impact of new energy vehicle high-voltage harness from the use characteristics of new energy vehicles, and defines the determination standard of SOD, which has positive significance for the design process control and product quality improvement of vehicle high-voltage harness system.

【关键词】DFMEA; 高压线束; 新能源汽车

【Keywords \DFMEA; high-voltage harness; new energy vehicles

【DOI】10.36012/peti.v1i2.864

\section{1 引言}

对于新能源汽车而言, 高压线束系统是新能源电动汽车 的关键零部件之一, 是保障电动车的安全可靠运行的关键零 部件。高压线束将高压电池、动力电机、电机控制器等各个高 压用电器连在一起 ${ }^{[1]}$ 。在同样的使用条件和工况下,与传统燃 油汽车相比, 由于高压线束承载高电压, 所以存在更多的安全 隐患, 为了降低高压线束系统在设计过程中的潜在隐患, 将 DFMEA 工具用于高压线束系统项目的设计中, 对高压线束系 统进行设计过程失效模式及影响分析, 明确严重 度 $\mathrm{S}$ 、频度 $\mathrm{O}$ 、探测度 $\mathrm{D}$ 判定标准, 以此来提升高 压线束系统设计质量。

\section{2 高压线束系统 DFMEA 思路}

DFMEA 全称 “Design Potential Failure Mode and Effective Analysis”, 中文翻译 “设计过程中潜 在的失效模式及其影响分析”。DFMEA 分析的主 要目的是识别设计过程中所有可能的失效模式, 并通过提出改进措施降低或消除潜在风险, 最终 达成产品的设计目标。DFMEA 是设计工程师必不 可少的设计过程风险管理工具, 从识别风险、评估 风险、消除风险三个方面贯穿整个设计过程 ${ }^{[2]}$ 。
对于 DFMEA 的编写, 首先需要收集项目历史问题, 从历 史问题数据库中寻找规律, 界定零部件之间的范围, 阐述系统 零部件之间的关系; 根据界定的范围得到系统边界图,再在边 界图之间识别干扰因子、错误因子和错误状态而得到 P 图; 最后依据 $\mathrm{P}$ 图分析高压线束的失效模式及其后果; 依据 DFMEA 的分析结果, 对失效模式的严重度、频度、探测度进行 评判, 最终得到失效模式 RPN 值 (风险优先数), 根据不同失 效模式的 RPN 值对失效模式进行风险评估和持续改进 ${ }^{[3]}$ 。图 1 为高压线束系统 DFMEA 编写思路。
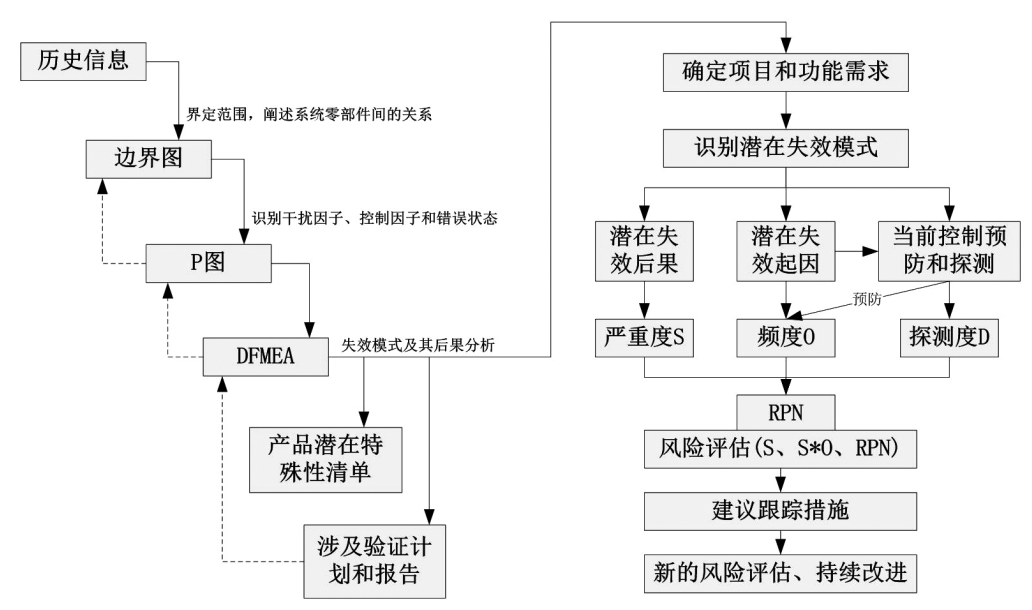

图 1 高压线束 DFMEA 编写思路 


\section{3 高压线束系统的边界图和 P 图}

对于新项目高压线束系统开发，往往会借助其他同类型 车型的相关问题进行前期问题排查, 从各部门收集信息, 界定 高压线束的系统影响边界; 问题来源主要是试制车间、质量 部、试验场、生产基地等部门。

对问题进行分类整理之后, 问题类型主要可以分为固定 高压线束不牢靠失效、与各高压用电器接口连接失效、高压线 束保护层失效、零部件个体差异失效、外部环境失效、随时间 的变化失效, 根据得到的失效类型, 界定高压线束系统失效边 界;图 2 高压线束系统边界图。

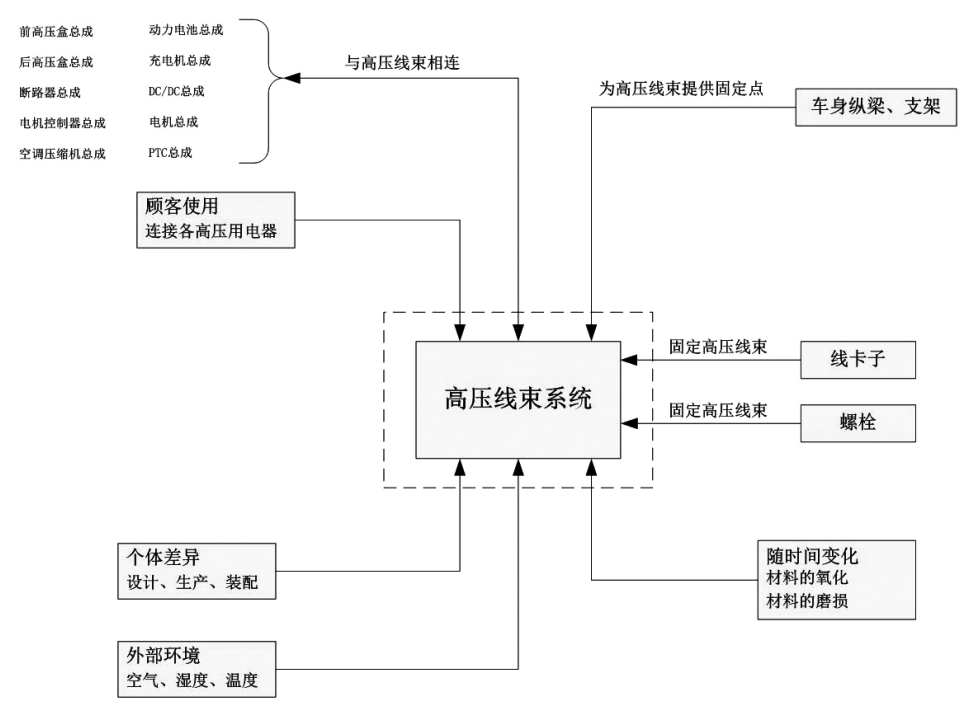

图 2 高压线束系统边界图

依据上述失效分类得到的高压线束系统失效边界图, 寻 找失效边界图中各部分之间的输入信号、干扰因子、控制因 子、错误状态及理想功能。

按照设计要求及各高压用电器需求, 与各高压用电 部件责任工程师召开 DFMEA 讨论会, 最终确定 P 图中各 项要求:

高压线束输入信号主要是安放正确的位置、与车身固 定、提供各被连接用电器的额定电流、最大工作电流及工作 温度等;

高压线束理想功能主要是实现高压用电器电源的可 靠连接、满足人员的安全防护安全、满足整车要求的相关 防护等级要求、满足总布置要求;

高压线束干扰因子主要是个体差异、内部环境、顾客 使用、外部环境及其他干扰因子; 个体差异干扰主要体现 在线缆材料的选择、制造工艺、制造精度、制造设备、装配 方式、存储运输方式等; 内部环境干扰主要体现在原材料
性能、安装方式、产品变形、特征面或线的控制、定位孔的选 择、型式试验的要求等; 顾客使用干扰主要是因为客户不正确 的操作使用方式; 外部环境干扰主要是因为车身焊接精度, 与 车体、前机舱、行李舱部件的距离, 离地间隙, 线束的安装方式 等方面; 除了上述干扰外, 还存在其他干扰, 如使用时间、保养 方式、天气、路况等干扰。

高压线束的控制因子主要是个体差异、内部环境、顾客使 用、外部环境及其他控制因子; 高压线束个体差异控制, 如选 择较好的制造工艺、保证制造精度、选择较好的制造工艺、保 证制造精度、选择成熟装配方式、确认运输方式; 内部环境的 控制主要是通过规定原材料性能、评审安装方式、控 制产品在运动过程中的变化、参考其他车型型式实验 要求; 顾客使用控制主要通过为顾客提供使用说明 书, 重要处粘占上说明和警告标识; 外部环境控制主 要通过控制车身上安装点的位置精度, 保证运动过程 中与其他部件不干涉; 其他干扰控制方式主要通过提 供较好的保养方式及保养阶段说明。

高压线束的错误状态主要是连接不可靠或者无 法实现连接、与其他部件发生干涉、无法满足整车防 护等级要求等; 图 3 高压线束系统 P 图。

\section{4 高压线束系统 SOD 判定标准}

基于高压线束 $\mathrm{P}$ 图得到的信息,对高压线束的严 重度 $(\mathrm{S})$ 、频度 $(\mathrm{O})$ 、探测度 $(D)$ 评分标准进行界定; 分 别对失效后果、失效发生的可能性、失效发生后的可 探测性进行分级, 每个维度分为十级, 对应得分为 1 10, 数字 越大表示对应的评判维度等级越高; 表 1 为严重度评价准则, 表 2 为频度评价准则, 表 3 为探测度评价准则。

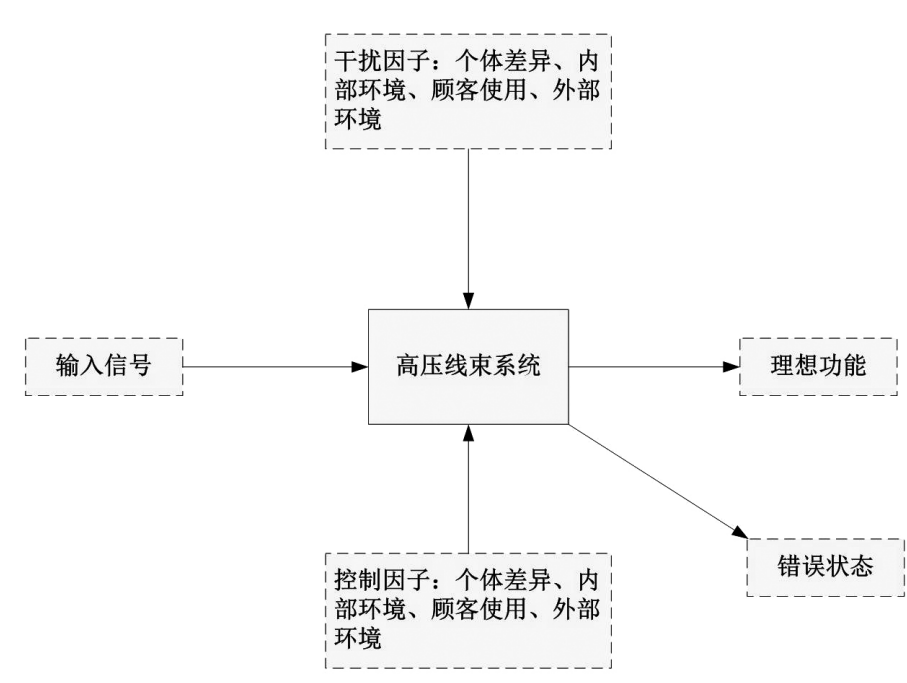

图 3 高压线束系统 $P$ 图 


\section{表 1 严重度评价准则}

\begin{tabular}{|c|c|c|}
\hline 后果 & 评定准则: 后果的严重度 & 严重度 \\
\hline $\begin{array}{l}\text { 无警告的 } \\
\text { 严重危害 }\end{array}$ & $\begin{array}{c}\text { 非常严重的失效模式,没有任何失效预警的情 } \\
\text { 况下影响到行车安全或不满足法规要求 }\end{array}$ & 10 \\
\hline $\begin{array}{l}\text { 有警告的 } \\
\text { 严重危害 }\end{array}$ & $\begin{array}{c}\text { 非常严重的失效模式,在有失效预警的情况下 } \\
\text { 影响到行车安全或不满足法规要求 }\end{array}$ & 9 \\
\hline 很高 & 车辆不能正常运行 & 8 \\
\hline 高 & 车辆可运行, 但性能下降, 顾客非常不满意 & 7 \\
\hline 中等 & $\begin{array}{c}\text { 车辆可运行, 但舒适性项目不可运行, 顾客不 } \\
\text { 满意 }\end{array}$ & 6 \\
\hline 低 & $\begin{array}{c}\text { 车辆可运行, 但舒适性项目的性能下降, 顾客 } \\
\text { 有些不满意 }\end{array}$ & 5 \\
\hline 很低 & $\begin{array}{c}\text { 配合和外观、异响等项目不舒服,75\%以上顾 } \\
\text { 客能感觉到有缺陷 }\end{array}$ & 4 \\
\hline 轻微 & $\begin{array}{c}\text { 配合和外观、异响等项目不舒服, 50\%的顾客 } \\
\text { 能感觉到有缺陷 }\end{array}$ & 3 \\
\hline 很轻微 & $\begin{array}{c}\text { 配合和外观、异响等项目不舒服, } 25 \% \text { 以下顾 } \\
\text { 客能感觉到有缺陷 }\end{array}$ & 2 \\
\hline 无 & 无明显可辨别或感知后果 & 1 \\
\hline
\end{tabular}

表 2 频度评价准则

\begin{tabular}{|c|c|c|}
\hline 失效发生的可能性 & 可能的失效率 & 频度数 \\
\hline \multirow{2}{*}{ 很高, 持续性失效 } & $>100$ 个, 每 1000 辆车/项目 & 10 \\
\hline & 50 个, 每 1000 辆车/项目 & 9 \\
\hline \multirow{2}{*}{ 高, 经常性失效 } & 20 个, 每 1000 辆车/项目 & 8 \\
\hline & 10 个, 每 1000 辆车/项目 & 7 \\
\hline \multirow{3}{*}{ 中等, 偶然性失效 } & 5 个, 每 1000 辆车/项目 & 6 \\
\hline & 2 个, 每 1000 辆车/项目 & 5 \\
\hline & 1 个, 每 1000 辆车/项目 & 4 \\
\hline \multirow{2}{*}{ 低, 相对很少发生的失效 } & 0.5 个, 每 1000 辆车/项目 & 3 \\
\hline & 0.1 个, 每 1000 辆车/项目 & 2 \\
\hline 极低, 失效不太可能发生 & $<0.01$ 个, 每 1000 辆车/项目 & 1 \\
\hline
\end{tabular}

表 3 探测度评价准则

\begin{tabular}{|c|c|c|}
\hline 探测度 & 评价准则: 设计控制可能探测出来的可能性 & 探测度 \\
\hline 绝对不可能 & $\begin{array}{c}\text { 设计控制将不能/或不可能找出潜在的 } \\
\text { 起因及失效模式 }\end{array}$ & 10 \\
\hline 很极少 & $\begin{array}{c}\text { 设计控制只有很极少的机会能找出潜在的 } \\
\text { 起因及失效模式 }\end{array}$ & 9 \\
\hline 极少 & $\begin{array}{c}\text { 设计控制只有极少的机会能找出潜在的 } \\
\text { 起因及失效模式 }\end{array}$ & 8 \\
\hline 很少 & $\begin{array}{c}\text { 设计控制只有很少的机会能找出潜在的 } \\
\text { 起因及失效模式 }\end{array}$ & 7 \\
\hline 少 & $\begin{array}{c}\text { 设计控制只有较少的机会能找出潜在的 } \\
\text { 起因及失效模式 }\end{array}$ & 6 \\
\hline 中等 & $\begin{array}{c}\text { 设计控制有中等机会能找出潜在的 } \\
\text { 起因及失效模式 }\end{array}$ & 5 \\
\hline 中上 & $\begin{array}{c}\text { 设计控制有中上多的机会能找出潜在的 } \\
\text { 起因及失效模式 }\end{array}$ & 4 \\
\hline 多 & $\begin{array}{c}\text { 设计控制有较多的机会能找出潜在的 } \\
\text { 起因及失效模式 }\end{array}$ & 3 \\
\hline 很多 & $\begin{array}{c}\text { 设计控制有很多的机会能找出潜在的 } \\
\text { 起因及失效模式 }\end{array}$ & 2 \\
\hline 几乎肯定 & 设计控制几乎能找出潜在的起因及失效模式 & 1 \\
\hline
\end{tabular}

\section{5 高压线束系统失效模式及影响分析}

在得到高压线束系统的边界图、P 图及失效模式的严重 度、频度、探测度的判定标准后, 可以对曾经出现过的或者将 来在设计开发过程中可能出现的故障模式进行分析, 综合分 析高压线束系统的失效模式及其影响; 根据 $\mathrm{P}$ 图确定输人信 号及理想功能, 对高压线束失效模式及其后果进行分析, 确定 每一种失效模式的 RPN 值, 根据 RPN 值对失效模式进行风 险评估并给出建议解决措施, 在设计阶段将该失效模式消除 或者将影响降低到可接受的范围之内; 表 4 为高压线束系统 的失效模式及影响分析。由于 DFMEA 篇幅过大, 此处仅展示 DFMEA 的表头及案例。

\section{表 4 高压线束系统的失效模式及影响分析}

\begin{tabular}{|c|c|}
\hline 项目 & 案例 \\
\hline 项目/功能 & 能正确和可靠地传输电源和信号 \\
\hline 潜在失效模式 & 虚接、脱落等接触失效 \\
\hline 潜在失效后果 & 功能缺失, 用电器不能正常工作 \\
\hline 严重度(S) & 8 \\
\hline 潜在失效起因/机理 & $\begin{array}{c}\text { 接插件接触不可靠, 接插件锁片锁止 } \\
\text { 不可靠 }\end{array}$ \\
\hline 频度(O) & 3 \\
\hline 现行设计控制预防 & $\begin{array}{c}\text { (1)从量产故障率低的接插件库中选用; } \\
\text { (2)参考对标车型接插件设计 }\end{array}$ \\
\hline 现行设计预防探测 & $\begin{array}{c}\text { 低压连接器应符合 QJD } 1920-2009 \text { 《汽车 } \\
\text { 插接件技术条件》要求, 高压接插件应符 } \\
\text { 合 SAE J1742-2010 }\end{array}$ \\
\hline 探测度(D) & 3 \\
\hline RPN & 72 \\
\hline 建议措施 & $\begin{array}{c}\text { 接插件试验结果分析; 根据试验结果调整 } \\
\text { 接插件方案 } \\
\end{array}$ \\
\hline
\end{tabular}

\section{6 结语}

DFMEA 是一个在产品设计开发阶段对开发质量进行全 面控制的一种可靠工具, 笔者基于与各部门的交流沟通, 历史 问题的整理, 按照 DFMEA 工具流程进行分析和评判, 可能存 在思考盲区; 在后续的新项目中, 需要对高压线束开发 DFMEA 进行持续改进与完善, 希望对本文读者的高压线束设 计开发工作有所参考借鉴。

\section{参考文献}

[1]GB/T 18384.1-2015 电动汽车安全要求第 1 部分: 车载储能 装置[S].

[2]胡庆华.DFMEA 在新产品开发中的有效应用[J]. 电子制作, 2015(4):78-79.

[3]Stamatis D H.Failure Mode and Effect Analysis FMEA from Theory to Execution( 故障模式影响分析 FMEA 从理论到实践)[M].陈 晓㶦,姚绍华,译.北京:国防工业出版社,2005. 